\title{
Neovascular glaucoma after central retinal vein occlusion in pre-existing glaucoma
}

\author{
Hsi-Fu Chen ${ }^{1}$, Min-Chi Chen ${ }^{3,4}$, Chi-Chun Lai ${ }^{2,4}$, Ling Yeung ${ }^{1,4}$, Nan-Kai Wang ${ }^{2,4}$, Henry Shen-Lih Chen ${ }^{2,4}$, \\ Wan-Chen $\mathrm{Ku}^{1}$, Shiu-Chen $\mathrm{Wu}^{2,4}$, Shirley H L Chang ${ }^{2,4}$ and Lan-Hsin Chuang ${ }^{1,4^{*}}$
}

\begin{abstract}
Background: To determine the outcome of central retinal vein occlusion (CRVO) in pre-existing glaucoma and the predisposing factors of developing neovascular glaucoma (NVG).

Methods: We retrospectively assessed a pre-existing glaucoma CRVO group and a non-glaucoma CRVO group to elucidate the demographics, clinical course and ocular parameters of these two cohorts. Among the pre-existing glaucoma cases, the predisposing factors for the development of NVG were monitored, including the retinal capillary non-perfusion status, intraocular pressure (IOP) and best-corrected visual acuity (BCVA) at presentation.

Results: Of 642 CRVO patients reviewed in this 10-year cohort study, 60 (9.3\%) had pre-existing glaucoma at a mean follow-up of 30.8 months, including 28 (4.4\%) primary open angle glaucoma (POAG), 27 (4.2\%) primary angle closure glaucoma (PACG), and $5(0.8 \%)$ normal tension glaucoma (NTG) cases. Although the presence of glaucoma in the CRVO eyes was not significantly associated with the risk of developing NVG, the incidence of developing NVG in pre-existing glaucoma eyes was significantly higher in the group with IOP greater than $20 \mathrm{mmHg}$ at CRVO presentation $(P=0.02$, Chi-square test) as well as in the ischemic CRVO group compared to the non-ischemic patients ( $P=0.005$, Fisher's exact test). Overall, $20 \%$ of pre-existing glaucoma patients needed glaucoma surgery after a CRVO event, including $11.7 \%$ of patients who developed iris neovascularisation (INV) and $8.3 \%$ of patients who developed a high IOP without INV.

Conclusions: Both the retinal non-perfusion status and uncontrolled IOP contribute to NVG in patients with pre-existing glaucoma after CRVO. Following CRVO, glaucoma surgery is necessary for pre-existing glaucoma cases with intractable elevated IOP with or without INV.
\end{abstract}

Keywords: Neovascular glaucoma, Central retinal vein occlusion with pre-existing glaucoma, Retinal non-perfusion status, Intraocular pressure, Predisposing factors

\section{Background}

Central retinal vein occlusion (CRVO) is a common retinal vascular disease that leads to visual impairment in elderly patients. Previous studies from Western countries have a high incidence of pre-existing primary open angle glaucoma (POAG) in patients with CRVO [1-3]. However, Asian populations differ from Western populations in that the incidence of primary angle closure glaucoma (PACG) is much higher. The incidence of different types of pre-existing glaucoma, intraocular

\footnotetext{
* Correspondence: c571019@cgmh.org.tw

'Department of Ophthalmology, Chang Gung Memorial Hospital, 222 Mai-Chin Rd, Keelung 204, Taiwan

${ }^{4}$ Chang Gung University College of Medicine, Taoyuan, Taiwan

Full list of author information is available at the end of the article
}

pressure (IOP) changes and optic neuropathy in CRVO in the Asian population is still unclear.

One-third of non-ischemic CRVO may convert to ischemic CRVO [4]. The major complication of ischemic CRVO is neovascular glaucoma (NVG) [4,5]. In CRVO patients with pre-existing glaucoma who have developed NVG, severe corneal oedema and recalcitrant high IOP are typically difficult to manage. Therefore, damage to the optic nerve is difficult to avoid, and any residual vision may be threatened. Thus far, it is still unknown whether pre-existing glaucoma plays a role in the development of NVG in CRVO patients. Regardless of treatment with intravitreal injection of

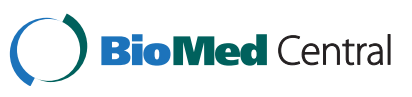

(c) 2014 Chen et al.; licensee BioMed Central Ltd. This is an Open Access article distributed under the terms of the Creative Commons Attribution License (http://creativecommons.org/licenses/by/4.0), which permits unrestricted use, distribution, and reproduction in any medium, provided the original work is properly credited. The Creative Commons Public Domain Dedication waiver (http://creativecommons.org/publicdomain/zero/1.0/) applies to the data made available in this article, unless otherwise stated. 
anti-vascular endothelial growth factor (VEGF) or corticosteroids, conventional panretinal photocoagulation (PRP) can regress retina neovascularisation in CRVO [6].

Because there is a paucity of evidence on this condition, the aim of this cohort study was to determine the profile of pre-existing glaucoma in CRVO in the East Asian population. Additionally, the secondary aims were to explore the predisposing factors and outcomes for the development of NVG in CRVO patients with pre-existing glaucoma.

\section{Methods}

This was a retrospective and comparative long-term cohort study. Patients were enrolled from Jul 1999 to Dec 2009. This study was performed in accordance with the Helsinki Declaration of 1975 (1983 revision) and was approved by the Institutional Review Board of the Chang Gung Memorial Hospital, Linkuo, Taiwan. All patients diagnosed with CRVO were identified and followed up monthly within one year, and their clinical data were recorded during a chart review.

Demographic characteristics, including gender, age, systemic diseases, the onset of CRVO and preceding eye history, were collected. The general ocular examination, including the best-corrected visual acuity (BVCA), slit-lamp biomicroscope examination, IOP at the first diagnosis of CRVO, indirect ophthalmoscopy, fluorescein angiography findings, or related surgery history, was documented in detail.

In this 10-year CRVO cohort study, the study group was consisted of pre-existing glaucoma cases diagnosed with increased IOP, an optic cup/disc ratio larger than 0.5 , and glaucomatous visual field changes. NVG was defined as neovascularisation of the iris with an IOP increase $\geq 21 \mathrm{mmHg}$. Optic nerve damage progression was defined as an increasing cup/disc ratio. The type of primary glaucoma, gonioscopy findings, optic cup/disc ratio and visual field data were also recorded. Newly diagnosed participants with CRVO were classified as the control group. We excluded patients who received intravitreous injection of anti-VEGF medications or triamcinolone acetonide during the follow-up period. As a proceeding analysis, patients who underwent vitrectomy to clear vitreous haemorrhages, patients who presented with distinct iris neovascularisation or neovascular glaucoma during the first visit or patients with significant diabetic retinopathy observed at short-term follow up (shorter than six months) were also precluded from this study. During the follow-up period, details regarding the procedures were also recorded for patients who underwent panretinal photocoagulation (PRP) or filtration surgery after CRVO.

Data were entered into Excel and were analysed using SPSS software (Statistical Package for Social Sciences).
The differences between the non-glaucoma and preexisting glaucoma groups were evaluated using a chisquare test and a 2-sample $t$-test for categorical and continuous variables, respectively. For the patients with pre-existing glaucoma, the relationships between the development of NVG and ocular parameters were examined using the Fisher's exact test or Wilcoxon rank sum test. Furthermore, the visual and optic nerve changes between the initial and final stages were assessed using the Wilcoxon signed-rank test. In this study, the Snellen visual acuity was converted to the logarithm of the minimum angle of resolution (logMAR) units for analysis. A $p$ value of less than 0.05 was considered significant.

\section{Results}

Overall, 642 patients with CRVO were reviewed in this 10-year cohort study. There were 60 eyes from 60 patients who had a history of pre-existing glaucoma before the onset of CRVO. The incidence of pre-existing glaucoma in CRVO was 60/642 (9.3\%). Regarding the various types of pre-existing glaucomas, there were 28 primary open angle glaucoma (POAG) cases, 27 primary angle closure glaucoma (PACG) cases and 5 normal tension glaucoma (NTG) cases in this study. A total of 321 patients were excluded from this study due to vitrectomy surgery or intravitreal injections of triamcinolone acetonide or anti-VEGF. To compare the characteristics, 60 pre-existing glaucoma cases were categorised as the study group, and 261 non-glaucoma cases were categorised as the control group.

The demographics, clinical characteristics and baseline ocular parameters are listed in Table 1 . In the preexisting glaucoma group, the age of CRVO onset was significantly older than the control group (57.3 vs. 67.2 years, $p<0.001)$. The prevalence of males was much higher than that of females in both groups. When comparing the ocular parameters, the cup/disc (C/D) ratio was substantially larger in the study group compared to the control group. According to the FAG finding, the proportion of ischemic type CRVO was higher in the pre-existing glaucoma group than in the control group (39.8\% VS $45 \%)$, although not significantly $(p=0.46)$. Moreover, during CRVO diagnosis, approximately one half $(48.3 \%)$ of the pre-existing glaucoma participants exhibited a higher increase in the IOP compared to the control group $(21.7 \pm 9.2$ vs. $13.9 \pm 4.8 \mathrm{mmHg}, p<0.001)$. However, the vision (BVCA) of both groups at the time of diagnosis was similar. There was no significant difference in the proportion of ischemic type CRVO between the two groups (Table 1). In general, $20.3 \%(53 / 261)$ of the non-pre-existing glaucoma cases and 31.7\% (19/60) of the pre-existing glaucoma cases developed NVG. $(p=0.06)$ Furthermore, the incidence of NVG occurred 
Table 1 Demographic and ocular characteristics of pre-existing glaucoma in central retinal vein occlusion (CRVO)

\begin{tabular}{|c|c|c|c|}
\hline & Non-glaucoma & Pre-existing glaucoma & $p$ value $e^{\dagger}$ \\
\hline Case number & 261 & 60 & \\
\hline Gender (female:male) & 102: 159 & $25: 35$ & 0.712 \\
\hline Age (mean $\pm S D)$ & $57.3 \pm 17.5$ & $67.2 \pm 14.9$ & $<0.001$ \\
\hline \multicolumn{4}{|l|}{ Systemic disease } \\
\hline DM & $71(27.2 \%)$ & $14(23.3 \%)$ & 0.540 \\
\hline HTN & $131(50.2 \%)$ & $29(48.3 \%)$ & 0.795 \\
\hline CRVO onset (months) (mean \pm SD) & $1.6 \pm 3.8$ & $1.7 \pm 3.6$ & 0.832 \\
\hline Perfusion status & & & 0.464 \\
\hline Non-ischemic & $157(60.2 \%)$ & $33(55 \%)$ & \\
\hline Ischemic & $104(39.8 \%)$ & $27(45 \%)$ & \\
\hline $\mathrm{C} / \mathrm{D}$ ratio $($ mean $\pm \mathrm{SD})$ & $0.42 \pm 0.09$ & $0.72 \pm 0.16$ & $<0.001$ \\
\hline IOP at presentation $(\mathrm{mmHg})($ mean $\pm \mathrm{SD})$ & $13.9 \pm 4.8$ & $21.7 \pm 9.2$ & $<0.001$ \\
\hline BCVA (logMAR) (mean \pm SD) & $1.46 \pm 0.85$ & $1.69 \pm 0.84$ & 0.053 \\
\hline PRP during follow up & $107(41 \%)$ & $22(36.7 \%)$ & 0.537 \\
\hline NVG during follow up & $53(20.3 \%)$ & 19(31.7\%) & 0.057 \\
\hline
\end{tabular}

Abbreviations: BCVA best-corrected visual acuity, C/D cup-to-disc, DM diabetes mellitus, HTN hypertension, IOP intraocular pressure, log MAR logarithm of the minimal angle of resolution, and SD standard deviation.

${ }^{+} p$ values were calculated using the chi-square test and 2-sample t-test for categorical and continuous variables, respectively.

within 6 months for ischemic CRVO was100\% (19/19) of cases in the pre-existing glaucoma group and $81.18 \%$ $(43 / 53)$ of cases in the control group $(p=0.05)$.

It is well known that the major complication after the onset of CRVO is the development of NVG, and IOP is typically intractable to medical treatment. To further identify the predisposing factors among the ocular parameters that contribute to the development of NVG in the study group, at the first visit, the IOP, C/D, and retinal nonperfusion status were obtained (Table 2). In the univariate analysis of the development of NVG, there was a significant difference in the mean IOP at the time of presentation between NVG within $0.5 \mathrm{yr}$ and nonNVG within $0.5 \mathrm{yr}(29.2 \pm 10.1$ vs. $19.8 \pm 8.29, P=0.007$, Wilcoxon rank sum test). Additionally, when the IOP was dichotomised as $>20$ or $\leqq 20 \mathrm{mmHg}, 10$ out of 32 (31.3\%) patients with IOP $>20 \mathrm{mmHg}$ and 2 out of 28 (7.1\%) with IOP $\leqq 20 \mathrm{mmHg}$ developed NVG within
0.5 yr, respectively (Chi-square test, $P=0.020$ ). Considering another fundamental parameter in the pre-existing glaucoma group that is associated with NVG development, a majority (93.3\%) of the patients had moderate to severe cupping upon the diagnosis of CRVO. The average C/D upon the presentation of CRVO was 0.72 in the study group. A high incidence of a large C/D ratio was found as $38.3 \%$ of the patients in the study group had moderate cupping $(0.5 \leqq \mathrm{C} / \mathrm{D}<0.8)$ and $55 \%$ had severe cupping ( $\geqq 0.8)$. For pre-existing glaucoma patients, there was a marginal significant difference in the $C / D$ ratio between NVG within $0.5 \mathrm{yr}$ and non-NVG within $0.5 \mathrm{yr}$ [0.78 \pm 0.17 , (range: $0.4-0.9$ ) vs. $0.70 \pm 0.16$, median 0.75 (range: 0.4-0.9) using the Wilcoxon rank sum test $(P=0.053)]$. During the study period of 30.8 months, C/D increased to subtotal cupping in 37 out of 60 (61.7\%) cases, indicating that the RNFL continued to thin, which is a serious consequence after retinal haemorrhage is

Table 2 NVG within 0.5 yr after CRVO in pre-existing glaucoma

\begin{tabular}{|c|c|c|c|}
\hline & $\begin{array}{l}\text { NVG } \\
\text { within } 0.5 \mathrm{yr}\end{array}$ & $\begin{array}{l}\text { Non-NVG } \\
\text { within } 0.5 y r\end{array}$ & $p$ value \\
\hline $\mathrm{C} / \mathrm{D}$ ratio at presentation (mean $\pm \mathrm{SD})$ & $0.78 \pm 0.17$ & $0.70 \pm 0.16$ & 0.053 \\
\hline Retinal perfusion status & & & $0.005^{*}$ \\
\hline Non-ischemic & 5 & 28 & \\
\hline Ischemic & 14 & 13 & \\
\hline Mean IOP at presentation $(\mathrm{mmHg})($ mean $\pm \mathrm{SD})$ & $29.2 \pm 10.1$ & $19.8 \pm 8.29$ & $0.007^{* *}$ \\
\hline $\mathrm{IOP}>20 \mathrm{mmHg}$ at presentation & 10 & 22 & \\
\hline $\mathrm{IOP} \leqq 20 \mathrm{mmHg}$ at presentation & 2 & 26 & $0.020^{\pi x \pi}$ \\
\hline
\end{tabular}

*Fisher's exact test; **Wilcoxon rank sum test; and ${ }^{* * *}$ Chi-square test. 
resolved. Furthermore, the incidence of developing NVG was significantly higher in the pre-existing glaucoma and control group patients with ischemic type CRVO compared to non-ischemic type CRVO. $(p=0.005$ and $p<0.001$, Fisher's exact test).

Accordingly, panretinal photocoagulation (PRP) was suggested in cases where neovascularisation of the iris occurred at follow-up. Nevertheless, there were 2 PACG cases with an initially normal IOP, which spiked after PRP was performed, and IOP stabilised with additional medication. Seventy-five percent of pre-existing glaucoma patients were regularly using anti-glaucoma agents before the first CRVO event. Among 27 pre-existing PACG patients, 9 patients had received laser iridotomy. Surgical interventions for NVG patients, including filtration surgery and transscleral cyclophotocoagulation, are summarised in Table 3. For patients with end stage glaucoma and poor vision, the type of surgery performed (trabeculectomy with mitomycin $\mathrm{C}$ or transscleral cyclophotocoagulation) depended on the physician's preference and patients' agreement. An intractable IOP increase without INV was treated with trabeculectomy with or without an antimetabolite agent if useful vision was still present. In this retrospective cohort study, 12 cases (20\%) needed surgical intervention to control IOP during the long-term follow up. The two major causes of surgery were uncontrolled IOP in cases that developed NVG (11.7\%) and intractable IOP without iris NV $(8.3 \%)$. It is worth noting that there were some preexisting glaucoma cases that flared up with high IOP without INV, and medical intervention was not sufficient thereafter.

Due to the retrospective nature of this study, the exact time point of conversion from the non-ischemic to ischemic type is difficult to detect. For example, a case of NTG with a BCVA of 0.6 with non-ischemic CRVO at presentation converted to ischemic CRVO that was subsequently complicated with NVG. This patient then hesitated PRP, and vision decreased to light sense negative. According to the records, 5 out of 33 non-ischemic cases in the pre-existing glaucoma group (15.1\%) developed NVG, and ischemic development was noted as possible for future development during the study period.
In general, vision did not significantly improve after conventional glaucoma management up to the 31-month follow-up. In patients with pre-existing glaucoma, vision deteriorated from an initial BCVA log MAR 1.69 (Snellen 0.02) to a final BCVA log MAR1.84 (Snellen 0.014). Because there are three types of pre-existing glaucoma, we analysed the visual outcome based on the mean BCVA and cup disc ratio change (Table 4). As indicated above and in Table 1, the C/D ratio for pre-existing glaucoma cases at CRVO presentation was larger than the control group ( 0.72 vs. 0.42$)$. When the initial and final ratios were compared, the $\mathrm{C} / \mathrm{D}$ ratio significantly increased in the overall, POAG, and PACG subgroups. This result demonstrates that the retinal nerve fibre layer defect continued to progress after CRVO, which may be associated with further deterioration of vision for these pre-existing glaucoma cases.

\section{Discussion}

Glaucoma is one of the known risk factors for CRVO $[1,2,7,8]$. A major complication of CRVO is the development of NVG, which can lead to severe visual impairment. Pre-existing glaucoma is seldom included in CRVO studies in Asian populations where the prevalence of PACG is higher than in the Western countries. Ethnicity may play an important role when evaluating ocular diseases related to glaucoma.

In this 10-year cohort study, the incidence of preexisting glaucoma in CRVO was similar to previous studies [7,9]. Recently, Michaelides and Foster also suggested that angle-closure may be associated with retinal vein occlusions in retrospective case series with diverse ethnicity [10]. In contrast, Hayreh et al. concluded that compartment syndrome contradicts the concept of playing a role in RVO [11]. In this study, it is notable that the incidence of PACG was approximately equal to POAG in CRVO. Regarding demographic and ocular parameters, CRVO was more prevalent in the elderly and middle to late stage glaucoma cases with large cupping. Although we do not have exfoliative glaucoma cases in our study, Prata et al. manifested that retinal vascular occlusion in patients with exfoliation syndrome occurs most frequently in the affected or more severely

Table 3 The management of various types of pre-existing glaucoma in central retinal vein occlusion (CRVO)

\begin{tabular}{|c|c|c|c|c|}
\hline & POAG & PACG & NTG & Overall incidence \\
\hline Case number & 28 & 27 & 5 & \\
\hline Prior use of antiglaucomaagents & $26(92.9 \%)$ & $16(26.7 \%)$ & $3(60 \%)$ & $75 \%$ \\
\hline Mean deviation $(\mathrm{dB})$ & -15.07 & -13.02 & -16.21 & -14.24 \\
\hline Trabe/TSCP for NVG & $1 / 3(14.3 \%)$ & $1 / 2(11.1 \%)$ & 0 & $11.7 \%$ \\
\hline Trabe for elevated IOP (w/o INV) & $2(7.1 \%)$ & $3(11.1 \%)$ & 0 & $8.3 \%$ \\
\hline
\end{tabular}

Abbreviations: PACG primary angle closure glaucoma, POAG primary open angle glaucoma, PRP pan-retinal photocoagulation, Trabe trabeculectomy, TSCP transscleralcyclophotocoagulation, and $w / o$ INV without iris neovascularisation. 
Table 4 Visual and optic nerve changesin various pre-existing glaucoma types

\begin{tabular}{|c|c|c|c|c|}
\hline & POAG & PACG & NTG & Overall \\
\hline Case number & 28 & 27 & 5 & \\
\hline IOP at presentation $(\mathrm{mmHg})$ & $24.3 \pm 10.0$ & $19.7 \pm 7.4$ & $18.6 \pm 11.3$ & $21.7 \pm 9.2$ \\
\hline Baseline BCVA (Snellen) & 0.027 & 0.015 & 0.023 & 0.02 \\
\hline Final BCVA (Snellen) & 0.018 & 0.013 & 0.005 & 0.014 \\
\hline \multicolumn{5}{|l|}{ Cup-to-disc ratio } \\
\hline Initial (mean $\pm S D$ ) & $0.69 \pm 0.17$ & $0.72 \pm 0.16$ & $0.88 \pm 0.04$ & $0.72 \pm 0.16$ \\
\hline Final $($ mean $\pm S D)$ & $0.75 \pm 0.16$ & $0.77 \pm 0.14$ & $0.88 \pm 0.04$ & $0.77 \pm 0.15$ \\
\hline$p$ value $^{\dagger}$ & 0.01 & 0.036 & & 0.001 \\
\hline Follow-up (months) (mean \pm SD) & $30.1 \pm 26.9$ & $32.3 \pm 32.8$ & $25.8 \pm 22.7$ & $30.8 \pm 29.1$ \\
\hline
\end{tabular}

Abbreviations: BCVA best-corrected visual acuity, NVG neovascular glaucoma, PACG primary angle closure glaucoma, POAG primary open angle glaucoma, and SD standard deviation.

${ }^{\dagger}$ Changes betweenthe initial and final cup-to-disc ratio were examined using the Wilcoxon signed-rank test.

affected eye [12]. This is in agreement with the consensus that large cupping in glaucoma patients is vulnerable to CRVO [3,12]. Moreover, the incidence of the ischemic CRVO type in pre-existing glaucoma was similar to that in the non-glaucoma population.

Beaumont et al. found that retinal venous occlusion at the optic cup has a higher IOP, C/D ratio and prevalence of POAG [3]. In contrast, Hayreh et al.compared CRVO patients and found that even without glaucoma medication, patients with pre-existing glaucoma had a lower IOP in the lesion eye compared to patients without preexisting glaucoma $[13,14]$. Cappin et al. found that the IOP was $4 \mathrm{mmHg}$ lower in the CRVO eye compared to the other eye in glaucoma patients. By iris angiography, they also found that the vessel of the iris was relatively dilated in CRVO eyes [15]. However, in the Taiwanese population, $46.7 \%$ and $45 \%$ of patients in the pre-existing glaucoma group were POAG and PACG, respectively. The IOP at CRVO presentation was higher in the pre-existing glaucoma group (mean: $21.7 \mathrm{mmHg}$ ) compared to the non-pre-existing glaucoma group $(13.9 \mathrm{mmHg})$. Because the mean diagnosed time was approximately 1.5 months in this study, it is unknown whether the IOP increase occurred before or after CRVO. The exact mechanism of IOP change after CRVO in different types of glaucoma requires further evaluation.

According to an evidence-based study on the natural course of CRVO, the proportion of non-ischemic cases that converted to ischemic cases was as high as $30 \%$ in three years [4]. The ischemic type CRVO can lead to NVG in $20 \%$ of patients [4]. In our study, for ischemic CRVO, there were more cases with NVG within 6 months in the pre-existing glaucoma group than the control group, but the number of cases was similar after longterm follow up. It is important to understand the other predisposing factors that contribute to the development of NVG in cases with primary glaucoma. We found that in patients with pre-existing glaucoma, the formation of
NVG within 0.5 yr was significantly, positively correlated with IOP at presentation and marginally correlated with the $C / D$ ratio at presentation. Due to the retrospective nature of this study, it is difficult to determine the exact IOP changes at the onset of CRVO. However, higher IOP or poor IOP control could theoretically compromise blood perfusion and increase the risk of neovascularisation of the iris. Therefore, it is important to periodically measure the IOP and control IOP after a CRVO event.

There was a similar incidence of the conventional management of PRP among the two groups, which suggests that pre-existing glaucoma is not prone to rubeosis iridis. According to the results of this study, the incidence of neovascular glaucoma with increased IOP was lower than the incidence of PRP performed for cases of rubeosis iridis. Although PRP was effective in eliminating ocular neovascularisation in CRVO in this study, Hayreh et al. revealed marked peripheral visual field loss after laser treatment [16]. One of the drawbacks of this retrospective study was the absence of visual field data collected after a CRVO event from; as a result, we could not conclude anything about the change in the visual field. Furthermore, a relatively high proportion (approximately 20\%) of preexisting glaucoma patients underwent glaucoma surgery after a CRVO event. This is the first study to address the consequence of two major causes of glaucoma surgery, NVG or persistent elevated IOP without ocular neovascularisation. The pathogenesis of intractable increased IOP without iris neovascularisation after CRVO is not clear. In previous reports, the levels of vascular endothelial growth factor were increased in the aqueous humour of glaucoma cases [17]. After a CRVO event, reduced blood flow in the retinal capillaries leads to hypoxia, upregulation and release of VEGF and ocular neovascularisation. Intractable IOP may be secondary to hypoxic and inflammatory changes after CRVO, all of which aggregated in the pre-existing glaucoma cases even though ocular neovascularisation did not develop. 
According to an epidemiology study, the severity of diabetic retinopathy and performance of PRP do not correlate with progressive enlargement of cupping [18]. In contrast, this study found that the ischemic change of the retina after CRVO in pre-existing POAG and PACG revealed a retinal nerve fibre layer defect, which progressed throughout the study period. Although with the limitation of the study that different clinicians observed the cupping, this study illustrates the change in the neuroretinal rim in CRVO with pre-existing glaucoma and may partially explain the reason for progressive deteriorated vision in these patients. Generally speaking, vision progressively deteriorated during the long-term follow up in this cohort study.

\section{Conclusions}

The distribution of pre-existing glaucoma in CRVO in the Taiwanese population is different from Western countries. Identifying the profile of pre-existing glaucoma in CRVO is fundamental to further identify effective management strategies. Both the retinal non-perfusion status and uncontrolled IOP contribute to NVG in patients with pre-existing glaucoma after CRVO. Physicians should be aware of the increased IOP after CRVO during follow-up, and glaucoma surgery is necessary for preexisting glaucoma cases with intractable elevated IOP with or without INV. For such underlying glaucoma diseases associated with glaucomatous optic nerve atrophy, prompt beneficial therapy to prevent complications and preserve vision requires further study.

\section{Competing interests}

The authors declare that they have no competing interests. There are no sources of financial support to declare in this paper.

\section{Authors' contributions}

HFC, LHC, MCC and CCL conceived of the study, participated in its design and helped to draft the manuscript. MCC and LHC also performed the statistical analysis. LY, NKW, HSLC, WCK, SCW, and SHL C participated in the data collection and analysis. All authors read and approved the final manuscript.

\section{Acknowledgments}

We do not have anyone or any sources of funding to acknowledge.

\section{Author details}

'Department of Ophthalmology, Chang Gung Memorial Hospital, 222 Mai-Chin Rd, Keelung 204, Taiwan. ${ }^{2}$ Department of Ophthalmology, Chang Gung Memorial Hospital, Linkuo Medical Center, Taoyuan, Taiwan. ${ }^{3}$ Department of Public Health and Biostatistics Consulting Center, School of Medicine, Chang Gung University, Taoyuan, Taiwan. ${ }^{4}$ Chang Gung University College of Medicine, Taoyuan, Taiwan.

Received: 6 May 2014 Accepted: 24 September 2014

Published: 5 October 2014

\section{References}

1. Soni KG, Woodhouse DF: Retinal vascular occlusion as a presenting feature of glaucoma simplex. Br J Ophthalmol 1971, 55:192-195.

2. Vadala' G, Zanini A, Favero C, Brogliatti B, Boles Carenini B: Evaluation of the clinical course of central retinal vein occlusion in eyes with and without glaucoma. Acta Ophthalmol Scand Suppl 1997, 224:16-17.
3. Beaumont PE, Kang HK: Cup-to-disc ratio, intraocular pressure, and primary open-angle glaucoma in retinal venous occlusion. Ophthalmology 2002, 109:282-286.

4. Central Vein Occlusion Study Group: Natural history and clinical management of central retinal vein occlusion. Arch Ophthalmol 1997, 115:486-491.

5. Mclntosh RL, Rogers SL, Lim L, Cheung N, Wang JJ, Mitchell P, Kowalski JW, Nguyen HP, Wong TY: Natural history of central retinal vein occlusion: an evidence-based systematic review. Ophthalmology 2010, 117:1113-1123.

6. The Central Vein Occlusion Study Group: A randomized clinical trial of early panretinal photocoagulation for ischemic central vein occlusion: the Central Vein Occlusion Study Group N report. Ophthalmology 1995, 102:1434-1444.

7. Koizumi H, Daniela C, Ferrara, Claudia B, Richard F, Spaide: Central retinal vein occlusion case-control Study. Am J Ophthalmol 2007, 144:858-863.

8. Sohan Singh H: Prevalent misconceptions about acute retinal vascularocclusivedisorders. Prog Retin Eye Res 2005, 24:493-519.

9. Moisseievl J, Howard D, Yael C, Ayala Lusky SM: Glaucoma and visualoutcome in central retinalveinocclusion. Acta Ophthalmol Scand 1996, 74:368-371.

10. Michaelides M, Foster PJ: Retinal vein occlusion and angle closure: a retrospective case series. J Glaucoma 2010, 19:643-649.

11. Hayreh SS, Zimmerman MB, Podhajsky PA: Retinal vein occlusion and the optic disk. Retina 2012, 2:2108-2118.

12. Prata TS, Rozenbaum I, de Moraes CG, Lima VC, Liebmann J, Ritch R: Retinal vascular occlusions occur more frequently in the more affected eye in exfoliation syndrome. Eye (Lond) 2010, 24:658-662.

13. Sohan Singh H, Bridget Z, Meena B, Patricia P: Intraocular pressure abnormalitiesassociated with central and hemicentral retinal vein occlusion. Ophthalmology 2004, 111:133-141.

14. Hayreh SS, March W, Phelps CD: Ocular hypotonyfollowingretinal vein occlusion. Arch Ophthalmol 1978, 96:827-833.

15. Cappin JM, Whitelocke R: The iris in central retinal thrombosis. Proc $R$ SoC Med 1974, 67:1048-1051

16. Hayreh SS, Klugman MR, Podhajsky P, Servais GE, Perkins ES: Argon laser panretinal photocoagulation in ischemic central retinal vein occlusion-a 10-year prospective study. Graefes Arch Clin Exp Ophthalmol 1990, 228:281-296.

17. Hu DN, Ritch R, Liebmann J, Liu Y, Cheng B, Hu MS: Vascular endothelial growth factor is increased in aqueous humor of glaucomatous eyes. J Glaucoma 2002, 11:406-410.

18. Klein BE, Moss SE, Magli YL, Klein R, Hoyer C, Johnson J: Optic disc cupping: prevalence findings from the WESDR. Invest Ophthalmol Vis Sci 1989, 30:304-309.

doi:10.1186/1471-2415-14-119

Cite this article as: Chen et al:: Neovascular glaucoma after central retinal vein occlusion in pre-existing glaucoma. BMC Ophthalmology 2014 14:119.

\section{Submit your next manuscript to BioMed Central and take full advantage of:}

- Convenient online submission

- Thorough peer review

- No space constraints or color figure charges

- Immediate publication on acceptance

- Inclusion in PubMed, CAS, Scopus and Google Scholar

- Research which is freely available for redistribution 